\title{
Physiology, pathophysiology, and clinical impact of claudins
}

\author{
Markus Bleich $^{1}$ (D) - Dorothee Günzel ${ }^{2}$
}

Received: 24 November 2016 / Accepted: 27 November 2016 /Published online: 6 December 2016

(C) Springer-Verlag Berlin Heidelberg 2016

\section{A special issue on claudins}

Life and disease of an organism are determined by homeostatic mechanisms which secure the composition of body fluids, temperature, and regulation of growth and metabolism. It also includes the control of interfaces between the organism and its environment. Here, epithelial function plays a central role. The layers of epithelial cells of outer and inner body surfaces serve two opposing goals: tightening and permeation. Barrier formation against pathogen invasion or loss of fluids and on the other hand simultaneous massive transepithelial transport of substrates, salts, and water require specific properties. Although the paracellular pathway has always been a part of our understanding of epithelial function [2], it became clear only recently that the perspective on its role changed from a passive barrier to an actively fine-tuned functional component of every epithelium. In addition, it became evident that either causes or symptoms of widespread diseases like cancer, dermatitis, nephrolithiasis, epithelial infections, and diarrhea emerge from a dysfunctional tight junctional proteome.

The paracellular pathway is regulated by tight junction strands in between adjacent cells, separating the luminal

Markus Bleich

m.bleich@physiologie.uni-kiel.de; http://www.physiologie.unikiel.de/

Dorothee Günzel

dorothee.guenzel@charite.de; http://klinphys.charite.de/

1 Physiologisches Institut der CAU, Olshausenstraße 40, 24098 Kiel, Germany

2 Institut für Klinische Physiologie, Charité, Campus Benjamin Franklin, Hindenburgdamm 30, 12203 Berlin, Germany from the basolateral compartment. The mesh of strands is connected by the tricellular junction at the edges of this two-dimensional network between the cells of an epithelial layer $[3,4]$. Molecular analysis of the paracellular pathway revealed several proteins which constitute the junction. Among these proteins, claudins [1] turned out to be the critical structural element of the tight junction as they are the determinants of selectivity, permeability, and tightness. But interestingly, claudins are more than this. They are determinants of cellular polarity and differentiation. Their expression correlates with epithelial integrity, proliferation, or cell death. Finally, claudins are components of signaling between environment and cells: beneficial for epithelial transport coupling or unfavorably misused as a docking station for hostile viruses and bacterial toxins to support pathogen invasion.

Concurrent to the discovery of claudins, several disease mechanisms could be pinned down to the dysfunction, dysregulation, or absence of claudins within the tight junction. Reminiscent of "channelopathies," which is a synonym for diseases associated with defective ion channels, a variety of diseases are associated with claudins and are awaiting to become "claudinopathies." And these diseases are obviously not only electrolyte disorders.

In this special issue, we want to advertise the exciting and wide ranging research field on claudin proteins and their function in health and disease. We invited young and senior scientists, all leading experts in the field, to give their perspectives and data on physiology, pathophysiology, and clinical impact of claudins. We address basic functional aspects for exemplary claudins in the kidney, the intestine, the inner ear, the lung, and for the peculiar blood-neuron barrier which is located between two internal compartments of the body. As claudins are not only involved in classical transport defects but become increasingly important in inflammation, host microbe 
interaction, and cancer, translational mining in this field already resulted in appealing solutions, e.g., for drug delivery.

Taken together, novel insights from claudin research will provide tools for diagnostics and treatment and a better understanding of the pathophysiology and clinics of "claudinopathies". In parallel, the field of tricellular junction research is emerging now with only short delay and will complete our understanding of the paracellular pathway in the near future.

We are deeply thankful to all the authors who offered their expertise and valuable time and contributed to this special issue on physiology, pathophysiology, and clinical impact of claudins.

\section{References}

1. Furuse M, Fujita K, Hiiragi T, Fujimoto K, Tsukita S (1998) Claudin1 and -2 : novel integral membrane proteins localizing at tight junctions with no sequence similarity to occludin. J Cell Biol 141:15391550

2. Greger R (1981) Cation selectivity of the isolated perfused cortical thick ascending limb of Henle's loop of rabbit kidney. Pflügers Arch 390:30-37

3. Menco BP (1988) Tight-junctional strands first appear in regions where three cells meet in differentiating olfactory epithelium: a freeze-fracture study. J Cell Sci 89(Pt 4):495-505

4. Staehelin LA (1973) Further observations on the fine structure of freeze-cleaved tight junctions. J Cell Sci 13:763-786 